\title{
Hepatopulmonary syndrome caused by hypothalamic obesity and nonalcoholic fatty liver disease after surgery for craniopharyngioma: a case
} report

\author{
Dai Jung, MD, \\ Go Hun Seo, MD, \\ Yoon-Myung Kim, MD, \\ Jin-Ho Choi, MD, \\ Han-Wook Yoo, MD, PhD
}

Department of Pediatrics, Asan Medical Center Children's Hospital, University of Ulsan College of Medicine, Seoul, Korea
Received: 6 September, 2017

Revised: 17 October, 2017

Accepted: 10 November, 2017

Address for correspondence: Han-Wook Yoo, MD, PhD

Department of Pediatrics, Asan Medical Center Children's Hospital, University of Ulsan College of Medicine, 88 Olympic-ro 43-gil, Songpa-gu, Seoul 05505, Korea

Tel: +82-2-3010-3374

Fax: $+82-2-473-3725$

E-mail: hwyoo@amc.seoul.kr

https://orcid.org/0000-0001-8615$186 X$
Hypothalamic obesity is often complicated in patients with craniopharyngioma due to hypothalamic damage by the tumor itself, treatment modalities, and associated multiple pituitary hormone deficiency. Hypothalamic obesity causes secondary diseases such as nonalcoholic fatty liver disease (NAFLD) and diabetes mellitus (DM). We report a 19-year-old female who was diagnosed with craniopharyngioma, developed hypothalamic obesity after tumor resection, and progressed to hepatopulmonary syndrome. She manifested NAFLD 1 year after tumor resection. Two years later, the craniopharyngioma recurred, and she underwent a second resection. Three years after her second operation, she was diagnosed with type 2 DM, after which she did not visit the outpatient clinic for 2 years and then suddenly reappeared with a weight loss of $25.8 \mathrm{~kg}$ that had occurred over 21 months. One month later, she presented to the Emergency Department with dyspnea. Laboratory findings revealed liver dysfunction and hypoxia with increased alveolar artery oxygen gradient. Liver biopsy showed portal hypertension and micronodular cirrhosis. Echocardiography and a lung perfusion scan demonstrated a right to left shunt. She was finally diagnosed with hepatopulmonary syndrome and is currently awaiting a donor for liver transplantation. Patients surviving craniopharyngioma need to be followed up carefully to detect signs of hypothalamic obesity and monitored for the development of other comorbidities such as DM, NAFLD, and hepatopulmonary syndrome.

Keywords: Craniopharyngioma, Hepatopulmonary syndrome, Hypothalamus, Nonalcoholic fatty liver disease, Obesity

\section{Introduction}

Craniopharyngioma, originating from Rathke's pouch, is one of the most common suprasellar tumors detected in children. ${ }^{1)}$ The hypothalamus can be damaged by the craniopharyngioma itself as well as by treatment modalities including surgery or radiotherapy. ${ }^{2)}$ As the hypothalamus plays a crucial role in the homeostasis of the synthesis of anterior pituitary hormones, ${ }^{3)}$ damage to the hypothalamus results in dysregulation of multiple pituitary hormones and subsequent complications such as hypothalamic obesity, nonalcoholic fatty liver disease (NAFLD), and diabetes mellitus (DM). Up to $52 \%$ of pediatric craniopharyngioma patients develop hypothalamic obesity after tumor resection and most patients manifest obesity-related morbidities and low quality of life. Treatment by lifestyle modification, pharmacotherapy, and bariatric surgery has been attempted, ${ }^{2)}$ however, no definitive solutions are available. 
NAFLD is now a well-known sequelae of hypothalamic obesity. ${ }^{4-7)}$ A recent study of craniopharyngioma patients with hypothalamic involvement showed a $50 \%$ incidence of NAFLD, ${ }^{5)}$ raising the need for liver function monitoring in patients with hypothalamic injuries. The frequencies of NAFLD and nonalcoholic steatohepatitis (NASH) are increasing in children, and a few studies have reported patients who developed hepatopulmonary syndrome after resection of pituitary tumors. ${ }^{6,8)}$ In a previous case report, a patient underwent a pituitary tumor resection and was diagnosed with NASH and hepatopulmonary syndrome with dyspnea and hypoxemia 4 years later and required liver transplanatation. ${ }^{8)}$ However, cases of hepatopulmonary syndrome are rare, and the prognosis and long-term survival remain uncertain.

We describe a case of hepatopulmonary syndrome complicated with hypothalamic obesity, NAFLD, and type 2 DM after craniopharyngioma resection.

\section{Case report}

A 11-year and 2-month-old girl came to the hospital because of bifrontal headache, nausea, and vomiting. She was suspected to have craniopharyngioma on brain magnetic resonance imaging (MRI) and underwent surgical resection with a ventriculoperitoneal shunt. At the age of 11 years and 2 months, she weighed $60 \mathrm{~kg}$ (2.15 standard deviation score [SDS]) and was $152 \mathrm{~cm}$ (1.03 SDS) tall with a body mass index (BMI) of 26 $\mathrm{kg} / \mathrm{m} 2$ (>97th percentile) and Tanner stage I. After resection, she was treated using a cyber gamma-knife ( 3 cycle) due to the presence of residual tumor. She developed multiple pituitary hormone deficiencies, which were treated with hormone replacement therapy including levothyroxine $100 \mu \mathrm{g}$ daily, hydrocortisone $6 \mathrm{mg} / \mathrm{m}^{2} /$ day, oral desmopressin $0.1 \mathrm{mg} 3$ times per day, and conjugated estrogen $0.625 \mathrm{mg}$ daily. During her regular hospital follow-up, laboratory findings demonstrated an elevated aspartate aminotransferase (AST) level of $144 \mathrm{IU} /$ $\mathrm{L}$, an alanine aminotransferase (ALT) level of $65 \mathrm{IU} / \mathrm{L}$, a total cholesterol level of $281 \mathrm{mg} / \mathrm{dL}$, and a triglyceride level of 425 $\mathrm{mg} / \mathrm{dL}$, with increased echogenicity on her liver ultrasound, indicating NAFLD.

After 2 years of tumor resection, increased size of residual tumor was found, suggesting recurred craniopharyngioma.
She was referred to our hospital. At the age of 13 years and 3 months, her height, weight, and BMI were $155.5 \mathrm{~cm}$ (-0.08 SDS), $65.7 \mathrm{~kg}$ (1.81 SDS), and $27 \mathrm{~kg} / \mathrm{m}^{2}$ (97th percentile), respectively. Her sexual maturity rating was Tanner stage I. Laboratory findings showed an AST of $103 \mathrm{IU} / \mathrm{L}$, an ALT of $104 \mathrm{IU} / \mathrm{L}$, a total cholesterol of $230 \mathrm{mg} / \mathrm{dL}$, a triglyceride level of $359 \mathrm{mg} /$ $\mathrm{dL}$, total protein of $7.0 \mathrm{~g} / \mathrm{dL}$, serum albumin level of $4.3 \mathrm{~g} / \mathrm{dL}$, sodium $140 \mathrm{mmol} / \mathrm{L}$, potassium $3.7 \mathrm{mmol} / \mathrm{L}$, and chloride of $106 \mathrm{mmol} / \mathrm{L}$. Serum insulin-like growth factor-1 (IGF-1) and IGF-1 binding protein were $67.2 \mathrm{ng} / \mathrm{mL}$ and $3,596 \mathrm{ng} / \mathrm{mL}$, respectively. She underwent a second tumor resection at our

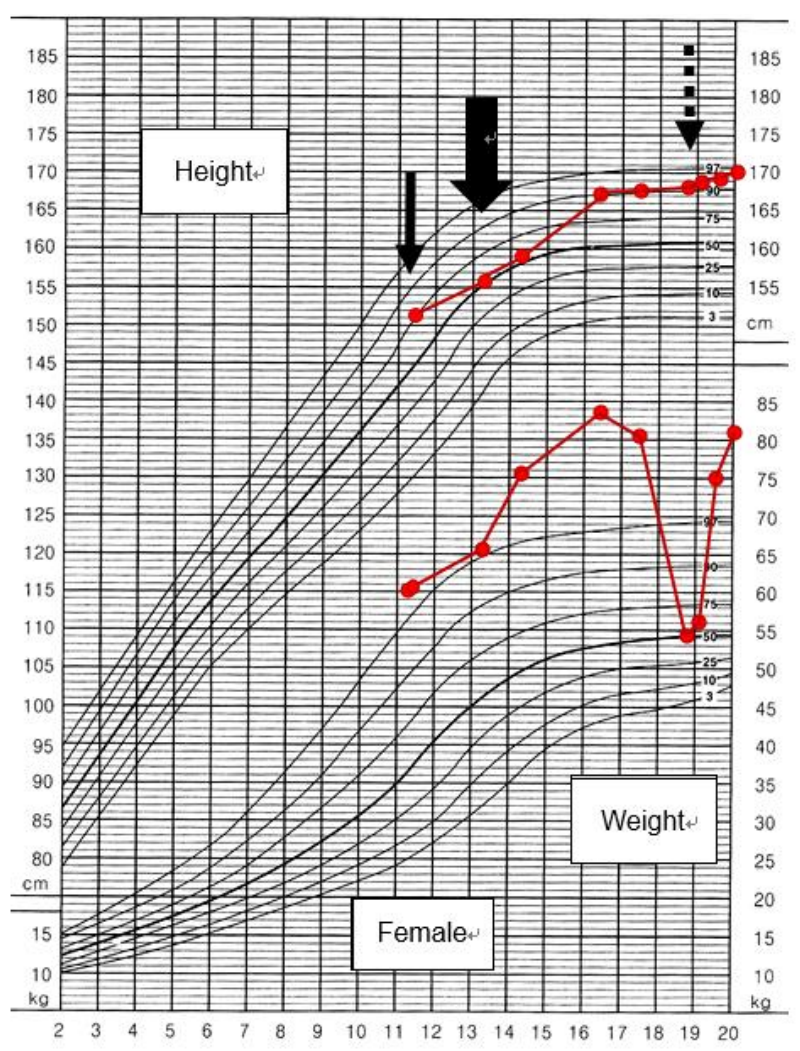

Fig. 1. The patient's growth curve during the follow-up period. The patient was diagnosed with craniopharyngioma and underwent tumor resection at the age of 11.2 years (plain arrow). However, the craniopharyngioma recurred and a second resection was performed at the age of 13.3 years (thick arrow). Hepatopulmonary syndrome occurred at the age of 18.9 years (dashed arrow).

Table 1. The patient's combined anterior pituitary function test after the second tumor resection

\begin{tabular}{lcc}
\hline Variable & Basal (normal range) & Peak (normal range) \\
\hline Growth hormone $(\mathrm{ng} / \mathrm{mL})$ & $0.1(1-5)$ & $0.1(>10)$ \\
Adrenocorticotropic hormone $(\mathrm{pg} / \mathrm{mL})$ & $11.9(0-60)$ & $23.5(>2-4$ fold of baseline) \\
Cortisol $(\mu \mathrm{g} / \mathrm{dL})$ & $0.48(5-25)$ & $0.38(>20)$ \\
Luteinizing hormone $(\mathrm{mlU} / \mathrm{mL})$ & $2.3(<2)$ & $3.6(>10)$ \\
Follicle-stimulating hormone $(\mathrm{mlU} / \mathrm{mL})$ & $0.1(<2)$ & $0.18(>2)$ \\
Thyroid stimulating hormone $(\mu \mathrm{U} / \mathrm{mL})$ & $0.22(0.5-6.5)$ & - \\
Free $T 4(\mathrm{ng} / \mathrm{dL})$ & $0.91(0.8-1.9)$ & - \\
Prolactin $(\mathrm{ng} / \mathrm{mL})$ & $6.8(0-15)$ & - \\
Estradiol $(\mathrm{pg} / \mathrm{mL})$ & $12.6(<14)$ & - \\
\hline
\end{tabular}


hospital. Two months later, she was diagnosed with multiple pituitary hormone deficiency on a combined anterior pituitary function test (Table 1) with Tanner stage I, and was kept on hormone replacement therapy. Three years after the second resection, her fasting blood glucose and hemoglobin $A_{1 c}$ levels were $126 \mathrm{mg} / \mathrm{dL}$ and $6.7 \%$, respectively. She was diagnosed with type $2 \mathrm{DM}$ associated with hypothalamic obesity, and was started on metformin. During the follow-up period, the NAFLD, dyslipidemia, and type 2 DM laboratory findings improved.

She was lost to follow up for 2 years and then showed up at the outpatient clinic at the age of 18 years and 9 months, with a weight loss of $25.8 \mathrm{~kg}$ that had occurred over 21 months. At this time, her height, weight, and BMI were $168.3 \mathrm{~cm}(1.44$ SDS), $54.3 \mathrm{~kg}$ (0.03 SDS), and $19.2 \mathrm{~kg} / \mathrm{m}^{2}$ (25th percentile), respectively showing Tanner stage of III (Fig. 1). One month later, she presented to the Emergency Department with dyspnea, orthopnea, anorexia, and general weakness. She had been not taking any medications for about 2 weeks. Her lips were cyanotic, her fingers clubbed, and her oxygen saturation was $88 \%$. Laboratory findings revealed thrombocytopenia (a platelet count of $59,000 / \mathrm{mm}^{3}$ ) and a prolonged prothrombin time (17 seconds; reference range, 10-13 seconds), indicating liver dysfunction. She was severely dehydrated with hypernatremia $(164 \mathrm{mmol} / \mathrm{L})$ and increased serum and urine osmolarity (346 mosm $/ \mathrm{kg}$ and $160 \mathrm{mosm} / \mathrm{kg}$, respectively). She was treated with intravenous fluids, oral desmopressin, and a stress dose of intravenous hydrocortisone. Mesenteric hepatic Doppler ultrasonography and dynamic computed tomography of the liver demonstrated a reduced liver volume with an engorged portal vein and splenomegaly. Her hepatic vein wedge pressure was $21 \mathrm{mmHg}$ (normal range, $1-5 \mathrm{mmHg}$ ) measured during transjugular liver biopsy, indicating portal hypertension.
The liver biopsy identified micronodular cirrhosis (Fig. 2). In room air, oxygen saturation was $88 \%$ and $94 \%$ in sitting and lying positions, respectively. Her alveolar arterial oxygen gradient was 77 , which was increased for her age (normal range, $5-10 \mathrm{mmHg}$ ). A bubble contrast echocardiography and lung perfusion scan suggested diffusely increased activity in the brain, kidneys, and spleen, which were consistent with a right to left shunt (Fig. 3). She was finally diagnosed with hepatopulmonary syndrome grade 3 due to liver cirrhosis induced by NAFLD, which was attributed to hypothalamic obesity after craniopharyngioma resection.

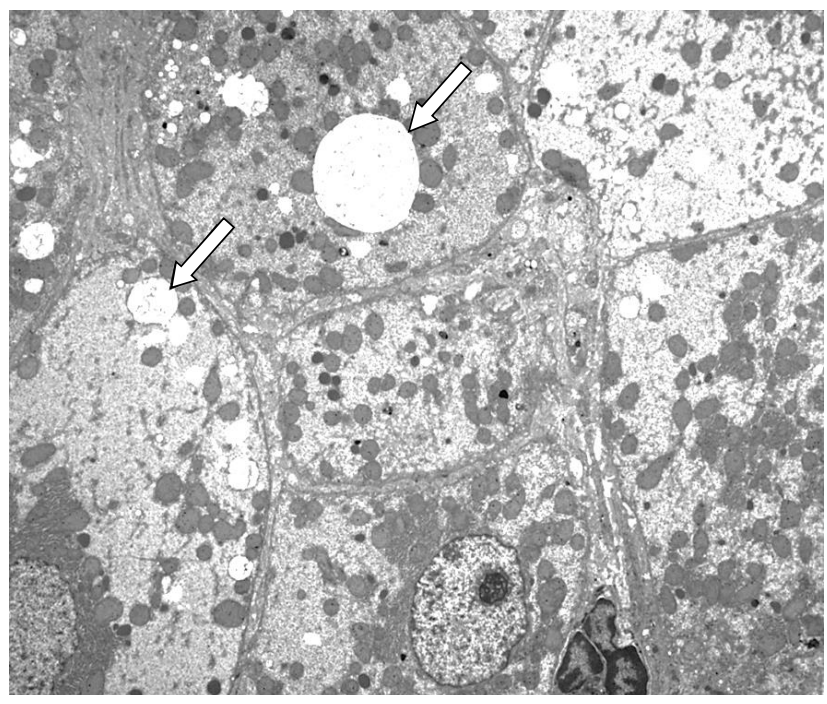

Fig. 2. Transmission electron microscopic image of a liver specimen. Pathological findings demonstrate micronodular cirrhosis with an increase in glycogen and cytoplasmic fat globules (arrow) $(\times 2,500)$.
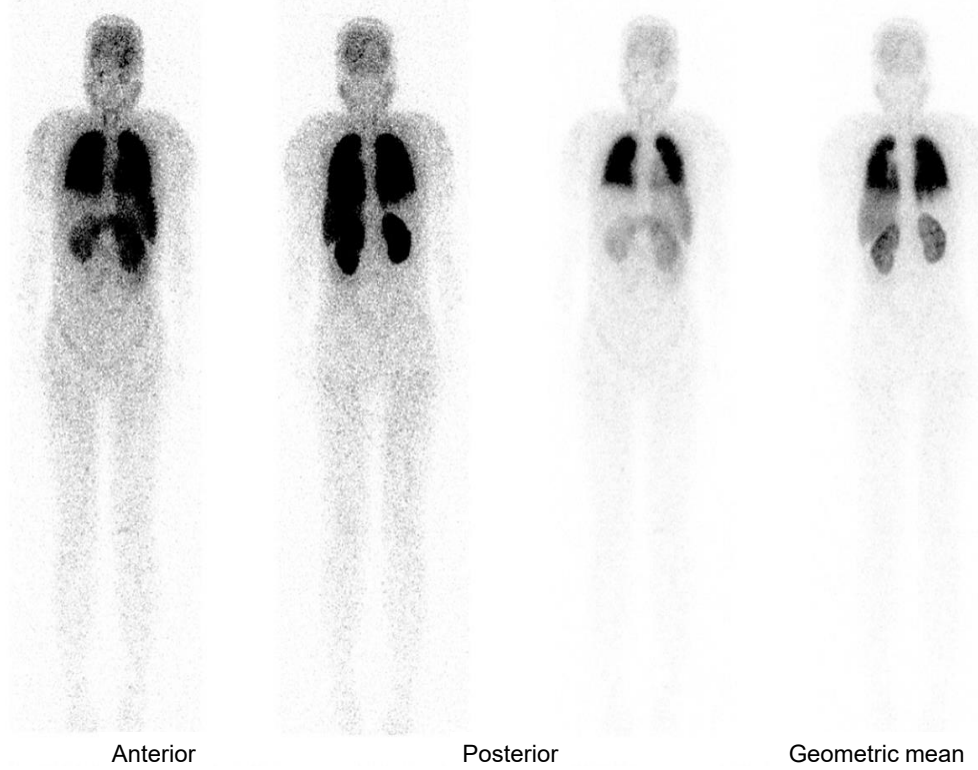

Fig. 3. Lung perfusion scan shows diffusely increased activity in the brain, kidneys, and spleen, indicating a right to left shunt. 
The patient was subsequently hospitalized three times because of aggravated dyspnea, revealing worsened grade 4 hepatopulmonary syndrome, dizziness with hypotension due to menorrhagia induced by thrombocytopenia, and hematemesis due to duodenal ulcer. She was followed up every 3 months with her prescribed hormone medications, including levothyroxine, prednisolone, desmopressin, recombinant human growth hormone, estrogen, and medroxyprogesterone acetate. At the age of 19 years, her height is $169.8 \mathrm{~cm}(1.72$ SDS) and weight is $60.6 \mathrm{~kg}(0.86 \mathrm{SDS})$ with a BMI of $21.0 \mathrm{~kg} / \mathrm{m}^{2}$ (25th-50th percentile) With metformin treatment, her hemoglobin $A_{1 c}$ level has decreased to $5.0 \%$, and her lipid profile has been normalized: total cholesterol $95 \mathrm{mg} / \mathrm{dL}$ and triglyceride $84 \mathrm{mg} / \mathrm{dL}$ (Table 2). Her AST and ALT levels have also been normalized, however, her prothrombin time is continuously prolonged, which is a sign of severe chronic cirrhosis of the liver. She has been registered as a candidate for cadaveric liver transplantation.

The study was waived from approval of the Institutional Review Board of Asan Medical Center has been waived, but informed consent was obtained from the patient.

\section{Discussion}

Hypothalamic obesity is a complication seen in patients with brain tumors, especially those that have undergone resection or radiation therapy that may have caused damage to the hypothalamus and pituitary gland.' In particular, hypothalamic obesity is found to be associated in $40 \%-50 \%$ of the patients with craniopharyngioma, and those with higher BMI at diagnosis are known to be at greater risk for the development of hypothalamic obesity. ${ }^{10,11)}$ Hypothalamic obesity not only decreases quality of life, but is also an important problem as it could be responsible for other comorbidities such as NAFLD and metabolic complications including hyperlipidemia and insulin resistance throughout the lifetime of an individual. ${ }^{5,12)}$ Our patient was diagnosed with NAFLD one year after and type 2 DM 5 years after her first tumor resection. Seven years after the development of NAFLD, she was diagnosed with hepatopulmonary syndrome.

Hypothalamic damage causes suppression of the sympathetic nervous system, resulting in compromised resting ability and voluntary energy expenditure. ${ }^{3)}$ Another pathophysiology of hypothalamic obesity is hyperinsulinemia and decreased insulin sensitivity caused by increased vagal efferent stimulation due to hypothalamic injury. ${ }^{13)}$ Serum leptin levels increase in hypothalamic obesity, suggesting leptin resistance. ${ }^{14)}$ Injuries in the ventromedial areas of the hypothalamus are known to be crucial factors in the manifestation of hyperphagia, ${ }^{15)}$ which has also been observed in animal studies. ${ }^{16}$

Patients with craniopharyngioma with hypothalamic involvement have been shown to have a low survival rate and severe weight gain during the follow-up period. The 10-year overall survival rate is $70 \%-92 \%$, and includes long term complications such as obesity, metabolic syndrome, NAFLD, and DM. In addition, quality of life decreases due to complicated sequelae. ${ }^{12}$ These findings indicate that patients with craniopharyngioma should be carefully monitored to detect signs of hypothalamic obesity and other secondary complications.

In a study of 21 patients with metabolic syndrome and obesity after hypothalamic damage, the mean onset of metabolic syndrome was 6.4 years after hypothalamic injury, and it was confirmed through liver biopsy that 10 patients had NAFLD. $\left.{ }^{6}\right)$ Two patients underwent liver transplantation, and one expired from recurrent NAFLD and hepatopulmonary syndrome after transplantation. ${ }^{6}$ Hepatopulmonary syndrome is a complication in patients with chronic liver disease. The pathogenesis remains to be discovered, but vascular dilatations induced by nitric oxide are suggested as the mechanism of hepatopulmonary syndrome. ${ }^{17}$ Vasodilation increases the pulmonary blood flow and cardiac output, subsequently leading to perfusion ventilation mismatch and arteriovenous shunts. In addition, it makes oxygen molecules hard to bind to red blood cell hemoglobins, resulting in hypoxemia. ${ }^{18)}$ Perfusion lung scan, contrast echocardiography, and pulmonary arteriography are used for diagnostic imaging modalities. Medical treatment is not very helpful and liver transplantation is considered to be the only available treatment. However, few case reports detail the recurrence of NAFLD despite successful liver transplantation. ${ }^{19,20)}$ This leads to a concern for patients who are diagnosed with hypothalamic obesity and secondary NAFLD progressing to cirrhosis of the liver, and liver transplantation should be cautiously considered.

In this study, we report a case of hepatopulmonary syndrome associated with NAFLD, dyslipidemia, and type 2 DM after hypothalamic damage due to craniopharyngioma. NAFLD led

Table 2. Serial laboratory findings of patient

\begin{tabular}{|c|c|c|c|c|c|c|c|c|}
\hline Age (yr) & AST (IU/L) & ALT (IU/L) & BUN (mg/dL) & Creatinine $(\mathrm{mg} / \mathrm{dL})$ & Cholesterol (mg/dL) & Triglyceride $(\mathrm{mg} / \mathrm{dL})$ & $\mathrm{HbA}_{1 \mathrm{c}}(\%)$ & Fasting glucose $(\mathrm{mg} / \mathrm{dL})$ \\
\hline 11 & 144 & 65 & - & 0.61 & 281 & 425 & 6.2 & 89 \\
\hline 13 & 103 & 104 & 9 & 0.6 & 211 & 359 & 5.8 & 111 \\
\hline 14 & 142 & 114 & 5 & 0.5 & 196 & 407 & 6.2 & 95 \\
\hline 15 & 46 & 43 & - & 0.6 & 172 & 314 & 6.0 & 131 \\
\hline 16 & 83 & 75 & 7 & 0.5 & 211 & 302 & 6.7 & 126 \\
\hline 17 & 77 & 40 & - & 0.5 & 137 & 98 & 5.7 & 83 \\
\hline 18 & 34 & 17 & 7 & 0.5 & 159 & 43 & 5.0 & 95 \\
\hline 19 & 27 & 11 & 8 & 0.59 & 113 & 123 & 5.5 & 82 \\
\hline
\end{tabular}

AST, aspartate aminotransferase; ALT, alanine aminotransferase; BUN, blood urea nitrogen; $\mathrm{HbA}_{1 c}$ glycosylated hemoglobin. 
to liver cirrhosis and portal hypertension, eventually leading to the development of hepatopulmonary syndrome. Patients with hypothalamic obesity should be carefully monitored for the early detection of rare fatal co-morbidities such as hepatopulmonary syndrome.

\section{Conflict of interest}

No potential conflict of interest relevant to this article was reported.

\section{Acknowledgments}

This work was supported by the Post-Genome Technology Development Program (10053626, Development of Decision Making System for Emergency Prediction) funded by the Ministry of Trade, Industry and Energy (MOTIE, Korea).

\section{References}

1. Rodriguez FJ, Scheithauer BW, Tsunoda S, Kovacs K, Vidal S, Piepgras DG. The spectrum of malignancy in craniopharyngioma. Am J Surg Pathol 2007;31:1020-8.

2. Page-Wilson G, Wardlaw SL, Khandji AG, Korner J. Hypothalamic obesity in patients with craniopharyngioma: treatment approaches and the emerging role of gastric bypass surgery. Pituitary 2012;15:84-92.

3. Lustig RH. Hypothalamic obesity after craniopharyngioma: mechanisms, diagnosis, and treatment. Front Endocrinol (Lausanne) 2011;2:60.

4. Hoffmann A, Müller HL. Novel perspectives on hypothalamic-pituitary dysfunction as a risk factor for nonalcoholic fatty liver disease. Minerva Endocrinol 2017;42: 132-44.

5. Hoffmann A, Bootsveld K, Gebhardt U, Daubenbüchel AM, Sterkenburg AS, Müller HL. Nonalcoholic fatty liver disease and fatigue in long-term survivors of childhood-onset craniopharyngioma. Eur J Endocrinol 2015; 173:389-97.

6. Adams LA, Feldstein A, Lindor KD, Angulo P. Nonalcoholic fatty liver disease among patients with hypothalamic and pituitary dysfunction. Hepatology 2004;39:909-14.

7. Bereket A, Kiess W, Lustig RH, Muller HL, Goldstone AP, Weiss R, et al. Hypothalamic obesity in children. Obes Rev 2012;13:780-98.

8. Fujio A, Kawagishi N, Echizenya T, Tokodai K, Nakanishi C, Miyagi S, et al. Long-term survival with growth hormone replacement after liver transplantation of pediatric nonalcoholic steatohepatitis complicating acquired hypopituitarism. Tohoku J Exp Med 2015;235:61-7.
9. Lustig RH, Post SR, Srivannaboon K, Rose SR, Danish RK, Burghen GA, et al. Risk factors for the development of obesity in children surviving brain tumors. J Clin Endocrinol Metab 2003;88:611-6.

10. Khan MJ, Humayun KN, Donaldson M, Ahmed SF, Shaikh MG. Longitudinal changes in body mass index in children with craniopharyngioma. Horm Res Paediatr 2014;82:3729.

11. Müller HL, Emser A, Faldum A, Bruhnken G, EtavardGorris N, Gebhardt U, et al. Longitudinal study on growth and body mass index before and after diagnosis of childhood craniopharyngioma. J Clin Endocrinol Metab 2004;89:3298-305.

12. Sterkenburg AS, Hoffmann A, Gebhardt U, Warmuth-Metz M, Daubenbüchel AM, Müller HL. Survival, hypothalamic obesity, and neuropsychological/psychosocial status after childhood-onset craniopharyngioma: newly reported longterm outcomes. Neuro Oncol 2015;17:1029-38.

13. Simoneau-Roy J, O'Gorman C, Pencharz P, Adeli K, Daneman D, Hamilton J. Insulin sensitivity and secretion in children and adolescents with hypothalamic obesity following treatment for craniopharyngioma. Clin Endocrinol (Oxf) 2010;72:364-70.

14. Roth C, Wilken B, Hanefeld F, Schröter W, Leonhardt U. Hyperphagia in children with craniopharyngioma is associated with hyperleptinaemia and a failure in the downregulation of appetite. Eur J Endocrinol 1998;138:8991.

15. Ahlskog JE, Randall PK, Hoebel BG. Hupothalamic hyperphagia: dissociation from hyperphagia following destruction of noradrenergic neurons. Science 1975; 190:399-401.

16. Albert DJ, Petrovic DM, Jonik RH, Walsh ML. Enhanced defensiveness and increased food motivation each contribute to aggression and success in food competition by rats with medial hypothalamic lesions. Physiol Behav 1991;49:13-9.

17. Fallon MB. Mechanisms of pulmonary vascular complications of liver disease: hepatopulmonary syndrome. J Clin Gastroenterol 2005;39(4 Suppl 2):S138-42.

18. Lv Y, Fan D. Hepatopulmonary syndrome. Dig Dis Sci 2015;60:1914-23.

19. Jankowska I, Socha P, Pawlowska J, Teisseyre M, Gliwicz D, Czubkowski P, et al. Recurrence of non-alcoholic steatohepatitis after liver transplantation in a 13-yr-old boy. Pediatr Transplant 2007;11:796-8.

20. Jonas MM, Krawczuk LE, Kim HB, Lillehei C, Perez-Atayde A. Rapid recurrence of nonalcoholic fatty liver disease after transplantation in a child with hypopituitarism and hepatopulmonary syndrome. Liver Transpl 2005;11:10810. 